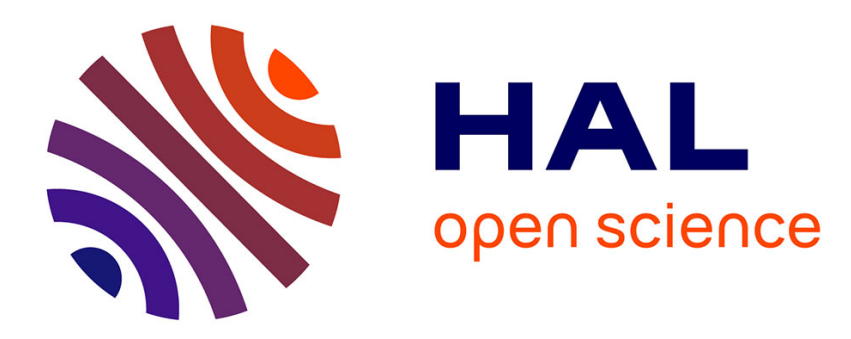

\title{
Michel Houellebecq: entre individualisme postmoderne et décadence fin de siècle
}

\author{
Jean-Michel Wittmann
}

\section{To cite this version:}

Jean-Michel Wittmann. Michel Houellebecq: entre individualisme postmoderne et décadence fin de siècle . Roman 20-50 : Revue d'étude du roman du XXe siècle, 2013, 56. hal-01697887

\section{HAL Id: hal-01697887 \\ https://hal.univ-lorraine.fr/hal-01697887}

Submitted on 31 Jan 2018

HAL is a multi-disciplinary open access archive for the deposit and dissemination of scientific research documents, whether they are published or not. The documents may come from teaching and research institutions in France or abroad, or from public or private research centers.
L'archive ouverte pluridisciplinaire $\mathbf{H A L}$, est destinée au dépôt et à la diffusion de documents scientifiques de niveau recherche, publiés ou non, émanant des établissements d'enseignement et de recherche français ou étrangers, des laboratoires publics ou privés. 


\section{Wittmann, Jean-Michel, «Michel Houellebecq : entre individualisme postmoderne et décadence fin de siècle », Roman $20 / 50 \mathrm{n}^{\circ} 56$, décembre 2013, p. 169-176.}

L'individualisme d'un côté, la décadence de l'autre, sont deux notions récurrentes dans les romans de Michel Houellebecq comme dans les études critiques qui leur sont consacrées, sans que le lien entre les deux soit nécessairement établi ni même clairement perçu. Il est vrai que le mot de décadence renvoie apparemment à l'ancrage des romans de Houellebecq dans la fin du XIX ${ }^{\mathrm{e}}$ siècle : il n'a jamais été aussi souvent prononcé qu'entre 1880 et 1914, dans une période à laquelle le romancier fait régulièrement référence, quoique de façon allusive. À l'inverse, son analyse de l'individualisme dans la société d'aujourd'hui témoigne de la double ambition - ambiguë pour cette raison même - d'être en phase avec les évolutions de son temps et d'en rendre compte : cette analyse s'inscrit en effet explicitement dans la lignée des réflexions d'un sociologue contemporain comme Gilles Lipovetsky, l'auteur de L'ère du vide. Essais sur l'individualisme contemporain (1989) ${ }^{1}$. Reste que la pensée de la décadence ou plutôt la représentation de la décadence, dans sa dimension politique, sociale et éthique, place en son cœur la question de l'individu et de son inscription problématique dans le groupe, à laquelle la littérature de la Belle Époque a largement contribué à donner corps. Dans ce contexte, la notion de décadence éclaire la réflexion sur l'individualisme proposée par Houellebecq, en révélant certaines représentations et certains topiques propres à un imaginaire de la décadence, qui contribuent à lester ses romans d'une idéologie essentiellement réactionnaire.

Encore cette notion de décadence se révèle-t-elle difficile à circonscrire, ce qui n'est sans doute pas pour déplaire à Houellebecq, dans la mesure où celui-ci ne cesse de multiplier dans ses romans des points de repères qu'il prend plaisir ensuite à esquiver ou à renverser. Pour les historiens de la littérature, le terme recouvre d'abord une éthique doublée d'une esthétique et il renvoie à un état d'esprit ou à un imaginaire qu'on rencontre déjà dans l'œuvre de Baudelaire, mais qui se fixe et s'épanouit à la fin du XIX ${ }^{\mathrm{e}}$ siècle, dans cette littérature qu'on dit précisément fin de siècle. Il n'est pas anodin que les écrivains représentatifs de cet état d'esprit ou de cet imaginaire soient fréquemment évoqués dans les romans de Houellebecq, ce qui n'a pas manqué d'attirer l'attention ${ }^{2}$. Mais lorsque dans Plateforme, le romancier évoque la foi des Européens dans «la supériorité de leur civilisation»pour conclure à leur décadence, il reprend plutôt à son compte certains des éléments constitutifs d'une antienne, celle de "l'éternelle décadence», analysée par un spécialiste de sciences politiques comme Michel Winock ${ }^{3}$ :

Cette conscience d'une mission civilisatrice s'était évaporée, tout au long du XIXe siècle. Les Européens, du moins certains d'entre eux, continuaient à travailler et parfois à travailler dur, mais ils le faisaient par intérêt, ou par attachement névrotique à leur tâche ; la conscience innocente de leur droit naturel à dominer le monde, et à orienter son histoire, avait disparu. Conséquence des efforts accumulés, l'Europe demeurait un continent riche; ces qualités d'intelligence et d'acharnement qu'avaient manifestées mes ancêtres, je les avais de toute évidence perdues. Européen aisé, je pouvais

\footnotetext{
${ }^{1}$ Voir notamment Sabine van Wesemael, «L’ère du vide », RiLUnE, n 1, 2005, p. 85-97, et Éric Fassin, «Le roman noir de la sexualité française », Critique $\mathrm{n}^{\circ}$ 637-638, juin-juillet 2000, p. 604-616), qui analyse notamment la «critique de l'individualisme sexuel » développée par Houellebecq.

${ }^{2}$ Voir Ieme Van der Poel, «Michel Houellebecq et l'esprit "fin de siècle" », in Sabine Van Wesemael (éd.), Michel Houellebecq, Amsterdam-New York, Rodopi, 2004, p. 47-54.

${ }^{3}$ Voir Michel Winock, Nationalisme, antisémitisme et fascisme en France, Paris, Seuil, coll. « Points », 1999, p. 99.
} 
acquérir à moindre prix, dans d'autres pays, de la nourriture, des services et des femmes ; Européen décadent, conscient de ma mort prochaine, et ayant pleinement accédé à l'égoïsme, je ne voyais aucune raison de m'en priver. $(307-308)^{4}$

De l'imaginaire de la décadence à la pensée de la décadence, il n’y a pourtant pas vraiment de solution de continuité, comme les travaux de Pierre Citti sur la littérature romanesque de la Belle Époque l'ont démontré 5 .

Il est frappant, au demeurant, mais sans doute pas accidentel, de voir que ces considérations sur le déclin de l'Occident aboutissent à présenter l' «Européen décadent » comme l'individu «ayant pleinement accédé à l'égoïsme», forme extrême de l'individualisme. Dès la fin du XIX ${ }^{\mathrm{e}}$ siècle, le lien direct entre le processus de la décadence et le développement de l'individualisme est notamment établi par Paul Bourget dans un texte qui a durablement marqué les esprits, "Théorie de la décadence», la partie centrale de son étude sur... Baudelaire, qui expliquait d'un même mouvement la décadence de l'œuvre d'art comme celle de la société à partir d'un principe unique, la prééminence de la partie sur le tout. Pour Bourget, la société «entre en décadence aussitôt que la vie individuelle s'est exagérée sous l'influence du bien-être acquis et de l'hérédité $»^{6}$. Afin d'étayer cette conclusion, il recourait à une allégorie lourde de sens, en présentant la société comme « un organisme » qui «se résout en en une fédération d'organismes moindres, qui se résolvent eux-mêmes en une fédération de cellules ». Chacune des parties doit donc se mettre au service du tout, comme chaque individu doit participer à la bonne marche de la société à laquelle sa propre survie est liée, étant donné que "Si l'énergie des cellules devient indépendante, les organismes qui composent l'organisme total cessent pareillement de subordonner leur énergie à l'énergie totale, et l'anarchie qui s'établit constitue la décadence de l'ensemble $»^{7}$.

Envisagée comme indissociable du développement de l'individualisme, sans pour autant lui être réductible, la question de la décadence renvoie à celle du lien social, elle-même présente comme un fil rouge dans les romans de Houellebecq. Dans l'univers du romancier, la montée de l'individualisme donne lieu à une description des caractéristiques de l'individu contemporain - parfaitement conforme à la symptomatologie établie par Lipovetsky - mais elle est aussi approchée comme un défaut de socialisation. Dans Extension du domaine de la lutte, le narrateur observe un «effacement progressif des relations humaines » $(48)^{8}$ en constatant par la même occasion que ce processus affecte la forme du roman contemporain, à la manière de Bourget qui établissait un lien entre décadence sociale et décadence esthétique. Il revient au psychiatre, dans le même roman, de s'interroger sur les « possibilités de rapports sociaux » offertes par le travail du narrateur, lequel serait pour le médecin « en quête de "repères d'identité" »(153-154). De même, la photo de Michel enfant, dans Les Particules élémentaires, rend compte d'une perspective de socialisation qui n'aura pas pu se concrétiser : « Il entrait dans le monde, il découvrait le monde, et le monde ne lui faisait pas peur; il se tenait prêt à prendre sa place dans la société des hommes. » $(280)^{9}$ Adulte, il est devenu un individu coupé du groupe, hanté par les penseurs et les écrivains du XIX ${ }^{\mathrm{e}}$ siècle, dont la vision

\footnotetext{
${ }^{4}$ Michel Houellebecq, Plateforme, Paris, Flammarion, 2001. Les références de pages entre parenthèses qui suivent les citations de ce roman dans cet article renverront systématiquement à cette édition.

${ }^{5}$ Voir notamment Contre la décadence. Histoire de l'imagination dans le roman (1890-1914), Paris, Presses Universitaires de France, 1987.

6 «Baudelaire»(«III. Théorie de la décadence »), in Essais de psychologie contemporaine [1883], rééd. Gallimard, coll. Tel n 233, 1993, p. 14.

${ }^{7}$ Ibid.

${ }^{8}$ Michel Houellebecq, Extension du domaine de la lutte, Paris, Maurice Nadeau, 1994. Les références de pages entre parenthèses qui suivent les citations de ce roman dans cet article renverront systématiquement à cette édition.

${ }^{9}$ Michel Houellebecq, Les Particules élémentaires, Paris, Flammarion, 1998. Les références de pages entre parenthèses qui suivent les citations de ce roman dans cet article renverront systématiquement à cette édition.
} 
pessimiste du monde est marquée par la problématique centrale du rapport entre l'organe et l'organisme, entre la partie et le tout : il lit par exemple, « assis sous un platane allée VictorCousin », le livre de Werner Heisenberg, «La Partie et le Tout, l'autobiographie scientifique de Werner Heisenberg » (il le «possédait depuis l'âge de dix-sept ans ») et condamne pour sa part la «mutation physique opérée par la science moderne», responsable de «l'individuation » et par-là même du malheur humain (28-29). Son frère Bruno, de son côté, est sensible à ce qu'il appelle, d'un terme contemporain, "l'idéologie du changement continuel », qui engage à "accepter que la vie d'un homme soit strictement réduite à son existence individuelle et que les générations passées et futures n'aient plus aucune importance à ses yeux » (210); mais ce processus n'est rien d'autre que ce qu'à la fin du XIX siècle, on désignait comme le déracinement, le terme ayant été mis à la mode par Barrès pour désigner la coupure de l'individu moderne avec ce qui le rattache à la collectivité, la terre, les morts, la tradition..., coupure elle-même responsable de la décadence dépeinte dans Le Roman de l'énergie nationale, qui, conformément au modèle défini par Bourget dans sa «Théorie de la décadence », résulte d'un développement excessif de l'organe (l'individu) au détriment de l'organisme (la société ou la nation, présentée par Barrès comme «dissociée et décérébrée $»^{10}$ ). Dans Plateforme, le narrateur, qui arrive difficilement à concevoir «qu'on soit attaché à une idée, à un pays, à autre chose en général qu'à un individu » (339), prend aussi conscience de son individualisme sur un mode négatif, en éprouvant l'absence de liens susceptibles de le rattacher à une communauté : «Je pris soudain conscience avec gêne que je considérais la société où je vivais à peu près comme un milieu naturel - disons une savane, ou une jungle - aux lois duquel j'aurais dû m'adapter. L'idée que j'étais solidaire de ce milieu ne m'avait jamais effleuré ; c'était comme une atrophie chez moi, une absence. Il n'était pas certain que la société puisse survivre très longtemps avec des individus dans mon genre [...]. » (339)

Dans ce contexte, il n'est pas étonnant de voir l'attention de Michel cristalliser autour de la notion de «sang», dans Les Particules élémentaires, même si précisément le personnage ne parvient pas à formuler clairement les idées, ou même seulement les questions, qu'il entrevoit ou qui l'attirent :

\begin{abstract}
Michel revint vers son bureau, inscrivit sur une feuille de papier : "Noter quelque chose sur le sang"; puis il s'allongea, éprouvant le besoin de réfléchir, mais il s'endormit presque aussitôt. Quelques jours plus tard il retrouva la feuille, inscrivit juste en dessous: "La loi du sang", et demeura perplexe une dizaine de minutes. (232)
\end{abstract}

Plus loin, Michel repense à la «phrase du Côté de Guermantes» qu'il a fait commenter par ses élèves et qui évoque «la pureté d'un sang où depuis plusieurs générations se rencontrait ce qu'il y a de plus grand dans l'histoire de France» (239), phrase qu'il va finalement renoncer à comprendre, en choisissant de retourner à Baudelaire. Cette image participe d'un système de pensée défini, ou mieux : d'une représentation cohérente de la société et de l'individu dont l'allégorie de la décadence de Bourget offre sans doute l'illustration la plus parlante, au même titre que la trilogie nationaliste de Barrès. Il n'est évidemment pas question ici d'établir une source, une influence, mais simplement de désigner le lien qui unit entre elle de façon logique diverses considérations chez Houellebecq, en repartant des textes écrivains qui ont repris l'antienne de la décadence à la fin du XIX ${ }^{\mathrm{e}}$ siècle, Bourget et Barrès et tête, qui n'hésitaient pas à désigner ce lien. L'individualisme, ou la décadence qui en résulte, se traduit par le fait que l'ensemble (l'organisme social) se dissocie à mesure que la partie (l'organe, l'individu) s'en désolidarise, courant elle-même vers sa propre perte : il s'agit donc d'une pathologie, dont la description permet cependant d'indiquer par la négative un idéal social.

\footnotetext{
10 «La France dissociée et décérébrée » est le titre du chapitre IX des Déracinés (1897), premier volet de la trilogie du Roman de l'énergie nationale.
} 
Derrière la maladie, en effet, se dessine en creux l'image de l'organisme social en bonne santé, caractérisé par son intégrité : organisme solidaire et clos, il doit préserver son équilibre propre et ne saurait s'ouvrir à des corps étrangers sans perdre du même coup son identité, sa santé, son intégrité.

Il n'est donc pas accidentel de voir resurgir ici et là, dans les romans de Houellebecq qui, précisément, exposent la situation de l'homme contemporain réduit à lui-même, victime d'un individualisme délétère dans une société occidentale présentée comme décadente, la métaphore de l'organisme social. C'est le cas dès Extension du domaine de la lutte, roman dans lequel les idées pour ne pas dire les poncifs de la décadence sont exposés par la voix de différents personnages, ce qui permet au romancier d'établir une distance ironique dans l'énonciation. De même que Jean-Pierre Buvet développe la thèse de l'épuisement vital (3637) dans la veine du Drieu la Rochelle des Notes pour comprendre le siècle (1941), obsédé par la décadence, ou que le psychiatre glose sur la «quête d'identité » déterminée par la perte des «liens sociaux » (153-154), Jean-Yves Fréhaut compare pour sa part « en quelque sorte la société à un cerveau, et les individus à autant de cellules cérébrales pour lesquelles il est en effet souhaitable d'établir le maximum d'interconnexions » (46). Il est frappant de voir le narrateur préciser alors : "Mais l'analogie s'arrêtait là. Car c'était un libéral, et il n'était guère partisan de ce qui est si nécessaire dans le cerveau : un projet d'unification. » (46) La représentation organiciste de la société repose en effet sur l'idée d'une parfaite coordination des organes qui composent le corps social, ce qui implique en réalité deux aspects complémentaires, l'interdépendance (les parties assurent la cohésion du tout qui, du coup, garantit leur pérennité) mais aussi la hiérarchisation (il revient au cerveau, c'est-à-dire à un pouvoir fort, de coordonner la bonne marche de l'ensemble et d'assurer sa cohésion). Si la question de la hiérarchisation n'est pas vraiment traitée dans les romans de Houellebecq, celle de l'interdépendance, ou plutôt celle de sa dissolution dans l'Occident moderne sous l'effet du développement de l'individualisme, est au centre de ses livres. C'est donc bien la cohésion de l'organisme social qui est centrale ici et qui détermine des réflexions apparemment plus ou moins dispersées sur l'identité collective.

La question de l'identité, soulevée de façon allusive et sibylline dans Les Particules élémentaires à travers la citation de Proust sur la «loi du sang », l'était déjà, de façon explicite, mais ironique, dans Extension du domaine de la lutte, à travers le diagnostic du psychiatre qui examine le narrateur:

L'épisode du voyage manqué dans l'Ardèche semble l'intéresser. En creusant un peu, il réussit à me faire avouer que mes parents étaient d'origine ardéchoise. Le voilà lancé sur une piste : d'après lui, je suis en quête de "repères d'identité". (153)

Un tel diagnostic pourrait tout à fait être désigné comme «barrésien » - le mal être du narrateur, archétype de l'individu contemporain, c'est bien le déracinement, d'autant que le psychiatre finit son intervention en s'interrogeant les «possibilités de liens sociaux » offerts au narrateur - et s'accorde à la représentation de l'individu et de la société représentée dans les romans de Houellebecq, sauf que ce dernier, suivant sa stratégie habituelle, met l'opinion à distance en la mettant au crédit d'un personnage comme le psychiatre.

L'allusion à la «loi du sang » traduit l'idée que, par delà le développement de l'individualisme, la perte d'intégrité fait courir un risque à l'organisme social, déterminant le tableau sombre proposé par les romans de Houellebecq. La décadence de la société moderne liée à l'éclatement des liens sociaux se traduit plus particulièrement dans Plateforme par la montée de la violence dans les banlieues $(259,276)$. Or Michel avoue ne comprendre «pas grand chose au monde moderne » qu'il associe aux «jeunes de banlieue » qui assurent le succès « de Nike, Adidas, Armani, Vuitton » et qui représentent l'un des « secteurs entiers de la société qui lui demeuraient étrangers » (281): la banlieue est associée à une modernité 
définie - négativement - par un multiculturalisme qui manifeste la perte d'intégrité et d'identité collective et qui est la version contemporaine du cosmopolitisme, dénoncé par les contempteurs de la décadence à la fin du XIX $^{\mathrm{e}}$ siècle. Or cette notion même de multiculturalisme est explicitement employée dans le roman, où elle est tournée en ridicule par le narrateur :

[...] un éditorial ridicule d'un certain Jacques Attali. Selon lui, la violence des jeunes des cités était un "appel au secours". Les vitrines de luxe des Champs-Élysées constituaient, écrivait-il, autant "d'étalages obscènes aux yeux de leur misère." Mais il ne fallait pas oublier que la banlieue était aussi "une mosaïque de peuples et de races, venus avec leurs traditions et leurs croyances pour forger de nouvelles cultures et pour réinventer l'art de vivre ensemble". Valérie me jeta un regard surpris : c'était bien la première fois que j'éclatais de rire en lisant L'Express.

Comme de juste, Houellebecq prend soin de laisser son discours flotter sur le plan idéologique, car la dénonciation d'Attali et du multiculturalisme fait suite à une charge contre Le Figaro glosant sur la montée de la violence dans les banlieues, afin de faire obstacle à la candidature de Lionel Jospin à l'Élysée (276). Il n'empêche que le rejet de cette mosaïque éclaire la rêverie sur la loi du sang et que le fantasme d'intégrité consubstantiel au discours sur la décadence de la fin de siècle trouve encore à s'exprimer dans des images qui, tout naturellement, filent la métaphore organiciste du corps social. Ainsi, le narrateur de Plateforme a « une espèce de vision sur les flux migratoires comme des vaisseaux sanguins qui traversaient l'Europe ; les musulmans apparaissaient comme des caillots qui se résorbaient lentement. » (30) : l'Occident décadent, qui pue «l'égoïsme, le masochisme et la mort » et a créé « un système dans lequel il est devenu simplement impossible de vivre » (369), est perçu et représenté comme un organisme malade, aux vaisseaux bouchés par les corps étrangers. De même, pour Bourget ou pour Barrès, la santé de l'organisme national, déjà éprouvée par l'individualisme, c'est-à-dire par la désolidarisation des organes, était mise à mal par des corps étrangers que Charles Maurras, dans un article célèbre, avait choisi de désigner comme les «quatre états confédérés » (Juifs, protestants, maçons et métèques) ${ }^{11}$. À l'inverse, l'image positive d'un individu réconcilié avec le milieu social auquel il appartient s'exprime dans celle d'un corps intègre, soudé autour d'une identité unique. Dans Les Particules élémentaires, au mariage de Bruno, Michel est frappé par les paroles du pasteur, qui explique : «Aimer sa femme, c'est s'aimer soi-même. Aucun homme n'a jamais haï sa propre chair, au contraire il la nourrit et il la soigne, comme fait Christ pour l'Église ; car nous sommes membres d'un même corps, nous sommes de sa chair et de ses os. » (214)

L'Occident décadent, menacé de l'intérieur par la montée de l'individualisme et de l'extérieur par un multiculturalisme qui le pénètre par les banlieues de ses grandes villes, pourrait-il être sauvé en se redécouvrant chrétien ? Le raccourci est tentant, mais Houellebecq lui-même prend soin de démystifier par avance toute conclusion de ce type au moment où il la suggère : dans Les Particules élémentaires, la dimension essentiellement réactionnaire d'un roman qui reprend à son compte les topiques majeurs du discours fin de siècle sur la décadence baigne dans un climat général de dérision, notamment avec l'irruption d'un Sollers présenté comme «un vrai clown gentil comme tout»: ce dernier se réjouit de ne trouver «pas trop d'influences » chez Michel qui «par exemple, n'est pas antisémite » (243), mais plus encore de le découvrir « réactionnaire », non sans ajouter "Mais il faut baiser, aussi, hein ? Il faut partouzer. C'est important. » (229) Plus généralement, l'usage systématique du second degré, des allusions culturelles volontairement disséminées dans le texte, la prise en compte de références idéologiques par différents personnages, tout contribue à instaurer un certain brouillage qu' on pourra juger moderne, voire postmoderne. Dans ce contexte, on peut

\footnotetext{
${ }^{11}$ Voir Charles Maurras, « Les quatre États confédérés », in L’Action française, 6 juillet 1912 ; repris dans Raoul Girardet, Le Nationalisme français. Anthologie, 1871-1914, Seuil, coll. Points / Histoire, 1983, p. 209-212.
} 
chercher le sens de cette représentation romanesque de la décadence d'une société occidentale minée par l'individualisme du côté de Jameson, pour qui «la décadence est clairement une chose qui, à la fois, résiste et vient après la modernité, comme un destin futur dans lequel toutes les promesses du moderne se distendent et se défont» et constitue «donc en quelque sorte la prémonition même du postmoderne $»^{12}$. Mais dans des romans qui ne cessent de mentionner Péguy, Schopenhauer etc, c'est bien la fin du XIX ${ }^{\mathrm{e}}$ siècle, hantée par le déclin et le spectre de la décadence qui donne l'arrière-plan de cette représentation pessimiste et contribue à lui donner du sens.

\footnotetext{
${ }^{12}$ Fredric Jameson, Le Postmodernisme ou la logique culturelle du capitalisme tardif, traduit de l'américain par Florence Nevoltry, Éditions Beaux-Arts de Paris, 2007, p. 527.
} 